ARTICLE

\title{
$\pi$ covalency in the halogen bond
}

\author{
Cameron W. Kellett (10) ${ }^{1}$, Pierre Kennepohl (i) ${ }^{1} \&$ Curtis P. Berlinguette (iD) $1,2,3,4 凶$
}

Halogen bonds are a highly directional class of intermolecular interactions widely employed in chemistry and chemical biology. This linear interaction is commonly viewed to be analogous to the hydrogen bond because hydrogen bonding models also intuitively describe the $\sigma$ symmetric component of halogen bonding. The possibility of $\pi$-covalency in a halogen bond is not contemplated in any known models. Here we present evidence of $\pi$-covalency being operative in halogen bonds formed between chloride and halogenated triphenylamine-based radical cations. We reach this conclusion through computational analysis of chlorine K-edge X-ray absorption spectra recorded on these halogen bonded pairs. In light of this result, we contend that halogen bonding is better described by analogy to metal coordination bonds rather than hydrogen bonds. Our revised description of the halogen bond suggests that these interactions could be employed to influence the electronic properties of conjugated molecules in unique ways.

\footnotetext{
${ }^{1}$ Department of Chemistry, The University of British Columbia, 2036 Main Mall, Vancouver, BC V6T 1Z1, Canada. ${ }^{2}$ Department of Chemical and Biological Engineering, The University of British Columbia, 2360 East Mall, Vancouver, BC V6T 1Z3, Canada. ${ }^{3}$ Stewart Blusson Quantum Matter Institute, The University of British Columbia, 2355 East Mall, Vancouver, BC V6T 1Z4, Canada. ${ }^{4}$ Canadian Institute for Advanced Research (CIFAR), 661 University

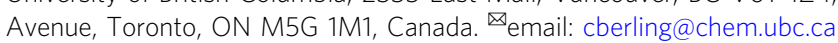


alogen bonding $(\mathrm{XB})$ is a strong intermolecular interaction that is commonly compared to hydrogen bonding $(\mathrm{HB})^{1-7}$. Both interactions are nearly linear attractions between a nucleophile (an HB or XB-acceptor) and an electrophilic site on the terminus of either a hydrogen or halogen atom bonded to an electron-withdrawing group (an $\mathrm{HB}$ or XB-donor, respectively $)^{5-8}$. Together, they comprise some of the strongest known intermolecular interactions ${ }^{6,9,10}$. Halogen bonding is widely used in many of the same applications as hydrogen bonding, including organic synthesis ${ }^{11}$, crystal engineering ${ }^{7,12}$, polymer engineering ${ }^{13}$, and medicinal chemistry ${ }^{14,15}$.

Halogen bonding is often described in the same terms as hydrogen bonding. The most commonly cited models of both interactions focus on the electrostatic attraction between the partial negative charge of a nucleophile with either the partial positive charge of a hydrogen atom or a small region of partial positive charge on the terminus of a halogen atom known as a $\sigma$ hole (Fig. 1) ${ }^{16-20}$. Other descriptions focus on the covalent charge-transfer component, where the lone pair of the nucleophile donates electron density into the hydrogen or halogen $\sigma^{*}$ antibonding orbital of the HB or XB-donor ${ }^{4,8,21-29}$. While both of these models have advantages and disadvantages, and an expansive view of these interactions will consider both as contributing factors ${ }^{7,21,24,30-33}$, they both describe hydrogen bonding and halogen bonding as the same type of interaction.

Despite these strong parallels between hydrogen bonding and halogen bonding, this analogy overlooks the possibility that halogen bonds can mediate not just $\sigma$-symmetric charge transfer, but also $\pi$-symmetric charge transfer (Fig. 1). While an HB-donor only has $\sigma$-symmetric orbitals on hydrogen, an XB-donor also has orbitals perpendicular to the $\sigma$-bond axis that are ideally oriented for $\pi$-symmetric orbital overlap. An intermolecular $\pi$-bond is therefore conceivable in cases where complementary low-energy empty $\pi^{*}$ orbitals and high-energy filled $\pi$ orbitals are present on the XB-donor and acceptor. As an example, consider a halogen bond formed with a $\pi$-donating nucleophile. In many if not most cases, a $\pi$-covalent interaction will not occur because all energetically accessible $\pi$ orbitals are filled (Supplementary Fig. 1a).
However, $\pi$-covalency is possible when low-energy empty $\pi$ orbitals on the XB-donor are made available, either by removing an electron from the filled $\pi$ orbital (Supplementary Fig. 1b) or by lowering the energy of the $\pi^{*}$ orbital (Supplementary Fig. 1c). The opposite situation, where $\pi$ backdonation occurs from the $\mathrm{XB}$-donor to the $\mathrm{XB}$-acceptor, is also plausible with appropriately positioned $\pi$ and $\pi^{*}$ orbitals.

We therefore propose that metal-ligand coordination bonds, where $\pi$-symmetric dative interactions are well known, make a better analogy for the nature of halogen bonding than hydrogen bonds. This shift in thinking has practical implications in addition to just rhetorical ones. It is well known that $\pi$-interactions in metal-ligand bonds have dramatic effects on the electronic properties of $\pi$-conjugated ligands. By extension, we believe that targeted design of halogen bonding pairs could leverage $\pi$ interactions to similarly impact the electronic properties of $\pi$ conjugated XB-donors and acceptors. Theoretical work by the Wong group has suggested that $\pi$ backdonation contributes to the strength of halogen bonds formed with nitroxide radical $\left(\mathrm{R}_{2} \mathrm{NO}^{\bullet}\right)$ nucleophiles ${ }^{34}$, but experimental validation and widespread discussion of the possibility of $\pi$-covalency in halogen bonds are completely absent in the literature.

We provide herein an analysis of experimental data that points to the clear existence of $\pi$-covalency in a halogen bond. We experimentally resolve this $\pi$-covalent component by studying a homologous pair of triphenylamine-based XB-donors denoted as Dye-X (where $\mathrm{X}=\mathrm{Br}$ or I), along with the non-halogen bonding analog, Dye-F (Fig. 2a) ${ }^{35-37}$. When oxidized by one electron to form the corresponding radical cation, the singly occupied molecular orbital (SOMO) of the open-shell Dye-X'+ is a $\pi$ symmetric MO with lobes oriented orthogonally to the halogen bond axis ${ }^{36}$. This SOMO is ideally positioned both spatially and energetically to accept electron density from a $\pi$-donating nucleophile as illustrated in Supplementary Fig. 1b. K-edge Xray absorption spectroscopy (XAS) is a powerful tool to investigate the electronic structure of metal-ligand bonds ${ }^{38-40}$. For halogen bonded dimers featuring a chloride nucleophile, it is possible to use chlorine XAS to probe the electronic structure of
Hydrogen bonding (HB)

Electrostatic model

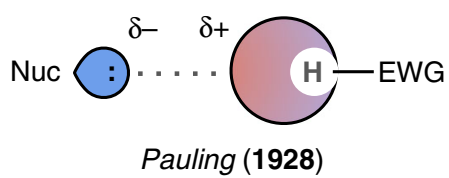

Orbital model

$\sigma$ interaction

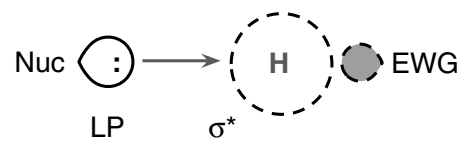

Pimentel and McClellan (1960)
Halogen bonding (XB)

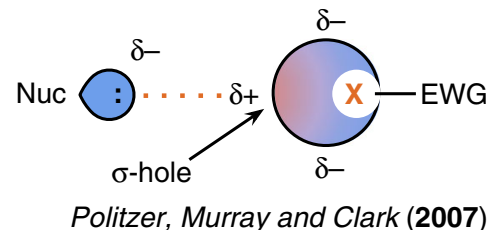

Politzer, Murray and Clark (2007)
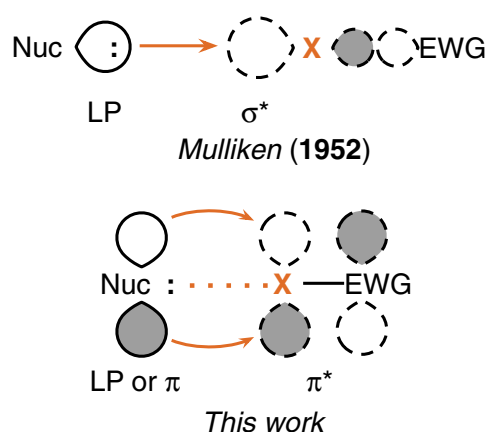

Fig. 1 Hydrogen bonding and halogen bonding models. Schematic representations of the electrostatic and covalent charge-transfer models of hydrogen bonding and halogen bonding 8,16,17,21,22,28,29. Our study shows that covalent $\pi$ bonding can also be operative in halogen bonds. 
a

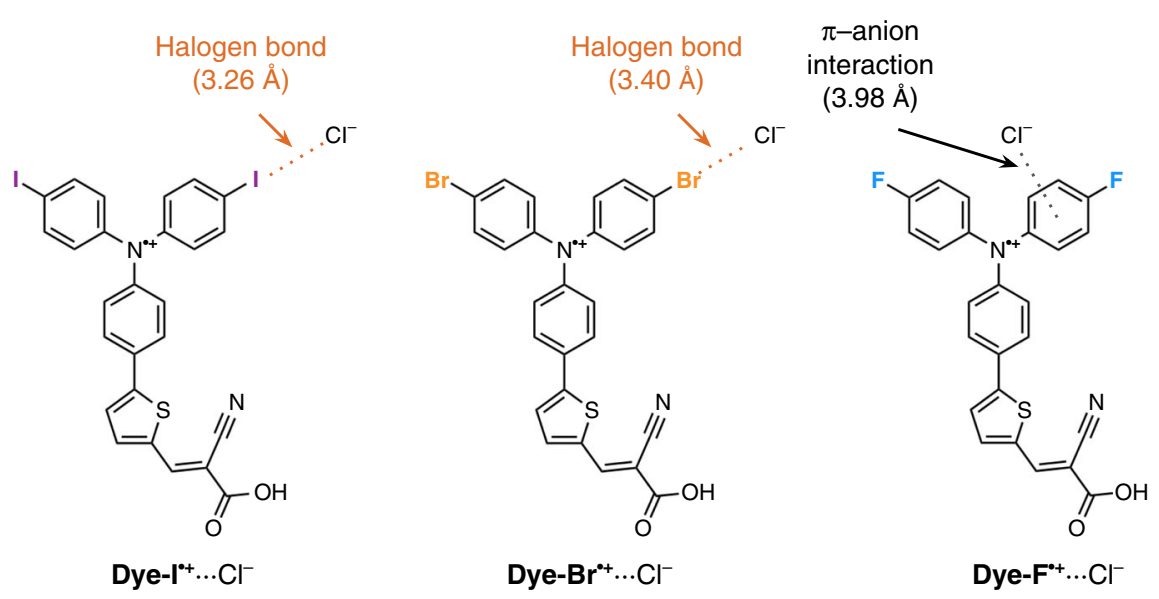

b
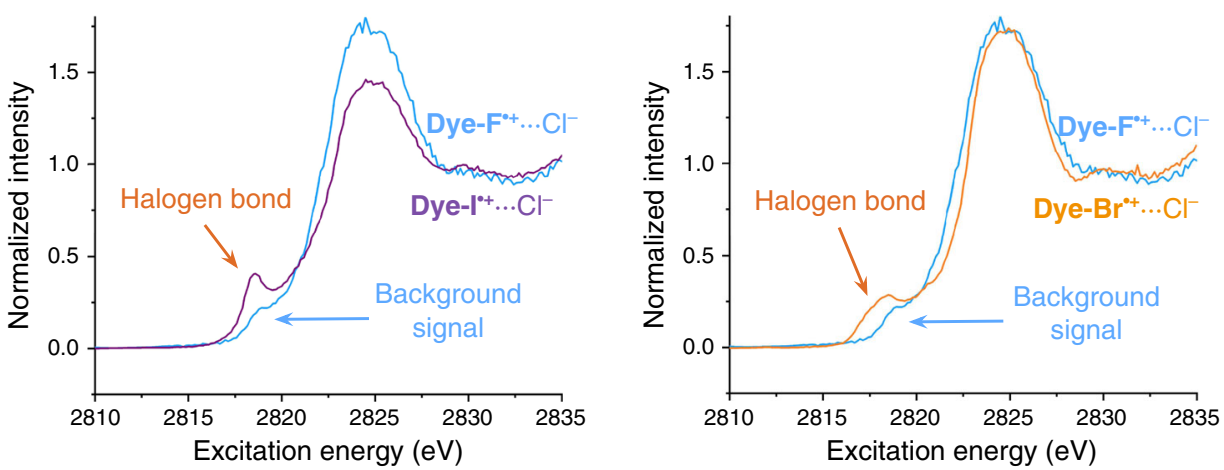

Fig. 2 Predicted adduct structures and experimental XAS spectra. a The Dye-X $\bullet+\ldots . \mathrm{Cl}^{-}$adduct structures predicted by DFT methods. $\mathbf{b}$ The experimental XAS spectra of the Dye- $\mathbf{I}^{+}+\ldots \mathrm{Cl}-$ halogen bonded dimer (purple line) and the $\mathbf{D y e}-\mathbf{B r}^{\bullet}+\ldots \mathrm{Cl}^{-}$halogen bonded dimer (orange line) overlaid with the nonhalogen bonding $\mathbf{D y e}-\mathbf{F}^{\bullet}+\ldots \mathrm{Cl}^{-}$control (blue line) ${ }^{37}$.

halogen bonds ${ }^{41,42}$. For the halogen bonded chloride adducts of Dye- $\mathbf{I}^{\bullet+}$ and Dye-Br ${ }^{\bullet+}$, we find that the well-resolved low-energy "pre-edge" spectral signature arises from $\pi$-orbital covalency.

\section{Results}

Experimental design. The Dye-X compounds consist of a redoxactive triphenylamine core decorated with halogen substituents and an anchoring group to adhere the compounds to a metal oxide surface (Fig. 2a). The triphenylamine moiety is not an electron-withdrawing group in the neutral form and consequently does not facilitate significant halogen bonding interactions. Oneelectron oxidation of the molecules to the corresponding radical cation states Dye-I'+ $\mathbf{I}^{+}$and Dye-Br ${ }^{\bullet+}$ greatly increases the electronegativity of the triphenylamine core and thereby promotes halogen bonding ${ }^{37}$. The triphenylamine core stabilizes the radical cation sufficiently to allow for spectroscopic studies ${ }^{43,44}$. The Dye-X compounds were adsorbed to a solid mesoporous $\mathrm{TiO}_{2}$ thin film with a common molecular orientation (Supplementary Fig. 2), thereby facilitating the study of halogen bonding interactions with a solution-phase species while suppressing selfinteraction between Dye-X molecules ${ }^{45}$. For our XAS experiments, the surface-anchored Dye-X compounds were oxidized with an acetonitrile solution of nitrosonium tetrafluoroborate $\left(\mathrm{NOBF}_{4}\right)$ then immersed in an acetonitrile solution of tetrabutylammonium chloride $\left(\mathrm{NBu}_{4} \mathrm{Cl}\right)$ and frozen at $77 \mathrm{~K}^{37}$.

Experimental chlorine $\mathrm{K}$-edge $\mathrm{X}$-ray absorption spectra. K-edge XAS directly probes the covalent interactions of individual elements in a molecular system by selectively exciting the core $1 \mathrm{~s}$ electrons with high-energy X-rays. This technique made it possible for us to isolate and selectively excite transitions in the Dye- $\mathbf{X}^{\bullet+}+. \mathrm{Cl}^{-}$dimers that involve only the nucleophilic chloride ions. A schematic describing the origins of the major XAS spectral features is presented in Supplementary Fig. 3. In the absence of this covalent charge-transfer interaction, the first available $\mathrm{Cl} \mathrm{K}$-edge XAS transition is the excitation from the $1 \mathrm{~s}$ shell into the empty $\mathrm{Cl} 4 \mathrm{p}$ orbitals of all chloride atoms present in the sample. This is observed as a very intense edge peak. Crucial to this study is the fact that a well-resolved pre-edge peak is observed if the valence $3 \mathrm{p}$ electrons of $\mathrm{a} \mathrm{Cl}^{-}$ion form a covalent interaction with the empty orbitals on the halogen of a Dye- $\mathbf{X}^{\bullet+}$ molecule. The integrated intensity of the pre-edge peak is directly proportional to the degree of orbital mixing in this covalent interaction 39,46 .

The experimental $\mathrm{Cl}$ K-edge XAS spectra of the halogen bonded Dye- $\mathbf{I}^{\bullet}+\ldots \mathrm{Cl}^{-}$and Dye- $\mathrm{Br}^{\bullet}+\ldots \mathrm{Cl}^{-}$dimers are shown in Fig. $2 b^{37}$. Three main spectroscopic features are observed prior to the edge jump at $>2828 \mathrm{eV}$ : A well-resolved pre-edge peak near $2818 \mathrm{eV}$; a pre-edge shoulder in the $2820-2 \mathrm{eV}$ range; and an intense edge peak between 2822 and $2828 \mathrm{eV}$. These features are highlighted using first and second derivative analyses in Supplementary Fig. 4. The pre-edge peak $(\sim 2818 \mathrm{eV})$ has a greater integrated area for Dye- $\mathrm{Br}^{\bullet}+\ldots \mathrm{Cl}^{-}$than for $\mathrm{Dye}-\mathrm{I}^{\bullet}+\ldots \mathrm{Cl}^{-}$, whilst the inverse is true of the pre-edge shoulder $(2820-2 \mathrm{eV})$. In our previous study, we assigned the well-resolved pre-edge peak as an excitation into the $\sigma^{*}$ orbital of the $\mathrm{C}-\mathrm{X}$ bond in accordance with the previously reported literature ${ }^{37}$. In this present study we will demonstrate that our preliminary assignment was indeed incorrect, and that the pre-edge transition is better described as an excitation into the $\pi^{*}$ orbitals of Dye- $\mathbf{X}^{\bullet+}$. 
The $\mathrm{Cl}$ K-edge XAS experiment does not discriminate between $\mathrm{Cl}$ atoms in different chemical environments, and therefore the observed spectra are a superposition of signals from all $\mathrm{Cl}$ atoms present in the sample. Moreover, the experimental XAS spectra in our previous study were collected in the presence of an excess of $\mathrm{Cl}^{-}$, and therefore the XAS signals arising from XB interactions will necessarily be convoluted by signals arising from other intermolecular interactions-most notably $\pi$-anion interactions between $\mathrm{Cl}^{-}$and the triphenylamine group of Dye- $\mathbf{X}^{\bullet+}$ - as well as interactions between the $\mathrm{Cl}^{-}$ions and the $\mathrm{TiO}_{2}$ substrate. The fluorinated equivalent of Dye- $\mathbf{X}^{\bullet+}\left(\mathbf{D y e}^{-F^{\bullet+}}\right)$ was therefore included in our previous study to control for these other interactions ${ }^{37}$. Without polarizable halogen substituents, Dye- $\mathrm{F}^{\bullet+}$ is not expected to engage in halogen bonding, but background signals arising from other $\mathrm{Cl}^{-}$interactions will still occur to approximately the same degree as in Dye- $\mathbf{B r}^{\bullet+} \ldots \mathrm{Cl}^{-}$and Dye- $\mathbf{I}^{\bullet}+\ldots \mathrm{Cl}^{-}$. In the Dye-F ${ }^{\bullet+} \ldots \mathrm{Cl}^{-}$XAS spectrum, only the edge peak between 2822 and $2828 \mathrm{eV}$ and a small pre-edge signal at $\sim 2819 \mathrm{eV}$ were observed. This small pre-edge feature was also observed in the spectrum of the blank $\mathrm{TiO}_{2}$ substrate treated with the $\mathrm{NOBF}_{4}$ oxidant $\left(\mathrm{TiO}_{2}{ }^{\mathrm{ox}}\right)$, and was therefore attributed to background $\mathrm{TiO}_{2}{ }^{\mathrm{ox}} \leftarrow \mathrm{Cl}^{-}$transitions arising from chloride interactions with $\mathrm{TiO}_{2}$ defect sites ${ }^{37}$. Because the pre-edge peak and pre-edge shoulder observed in the Dye- $\mathbf{I}^{\bullet}+\ldots \mathrm{Cl}^{-} / \mathbf{D y e}-\mathrm{Br}^{\bullet}+\ldots \mathrm{Cl}^{-}$ XAS spectra are absent in the Dye- $\mathrm{F}^{\bullet}+\ldots \mathrm{Cl}^{-}$spectrum, we can reliably attribute these features to halogen bonding interactions (Fig. 2b, Supplementary Fig. 4).

X-ray transition assignments. We undertook DFT simulations to define the chemical origins of the observed XAS transitions. The M06-2X functional has proven effective in describing bond lengths for halogen bonding interactions and was therefore used to generate geometry-optimized models of the Dye- $\mathrm{X}^{\bullet+}{ }^{+} . \mathrm{Cl}^{-}$ dimers $^{18}$. These models describe halogen bond distances of $3.26 \AA$ and $3.40 \AA$ for Dye-I ${ }^{\bullet}+\ldots \mathrm{Cl}^{-}$and Dye- $\mathrm{Br}^{\bullet}+\ldots \mathrm{Cl}^{-}$, respectively (Fig. 2a). As expected, optimization of the Dye-F ${ }^{\bullet}+\ldots \mathrm{Cl}^{-}$control did not place $\mathrm{Cl}^{-}$in a halogen bonding geometry, and instead positioned it $3.98 \AA$ above the face of a phenyl ring of Dye-F ${ }^{\bullet+}$, consistent with a weak $\pi$-anion interaction rather than a halogen bonding interaction (Fig. 2a). While it is expected that these $\pi$-anion interactions will have approximately the same XAS signal for all three Dye- $\mathbf{X}^{\bullet+}$ compounds, we nonetheless prepared geometry-optimized models of Dye- $\mathbf{I}^{\bullet}+\ldots \mathrm{Cl}^{-}$and Dye-Br ${ }^{*}+\ldots$ $\mathrm{Cl}^{-}$with chloride interacting with a phenyl group similar to Dye$\mathbf{F}^{\bullet+} \ldots \mathrm{Cl}^{-}$. These models are denoted Dye- $\mathbf{I}^{\bullet}+\ldots \mathrm{Cl}^{-}$-anion and Dye$\mathrm{Br}^{\circ}+\ldots \mathrm{Cl}^{-}$-anion and position the chloride anion $3.96 \AA$ and $4.00 \AA$ above the face of a phenyl ring, respectively.

Time-dependant DFT (TD-DFT) simulations of the XAS spectra were performed by calculating transitions originating from the chloride $1 \mathrm{~s}$ orbitals of the geometry-optimized models. Several DFT functionals with varying amounts of exact Hartree-Fock exchange were employed for these calculations as described in the Supplementary Methods section. Three distinct groups of transitions are predicted on the basis of the TD-DFT results for Dye-I ${ }^{\bullet}+\ldots \mathrm{Cl}^{-}$and Dye- $\mathbf{B r}^{\bullet}+\ldots \mathrm{Cl}^{-}$(Supplementary Figs. 5 and 6, respectively). These three groupings are easily visualized with the TD-DFT results calculated with the pure generalized gradient approximation (GGA) functional BP86 (Fig. 3): (i) a transition into the half-filled $\pi$ orbital of Dye- $\mathbf{I}^{\bullet+}$ or Dye-Br ${ }^{\bullet+}$ with significant $\pi$-orbital contributions from $\mathrm{Cl}^{-}\left(\pi^{*} \leftarrow \mathrm{Cl}_{1 \mathrm{~s}}\right)$; (ii) multiple transitions into a $\sigma^{*}$ orbital on Dye-I $\mathbf{I}^{+}$or Dye-Br ${ }^{\bullet+}$ involving the 3 p orbitals of $\mathrm{Cl}^{-}\left(\sigma^{*} \leftarrow\right.$ $\mathrm{Cl}_{1 \mathrm{~s}}$ ); and, (iii) a cluster of many transitions from the $\mathrm{Cl}^{-} 1 \mathrm{~s}$ orbital to an orbital with mixed Cl $4 \mathrm{p}$ and Dye- $\mathbf{I}^{\bullet+}$ or Dye-Br ${ }^{\bullet+}$ $\pi^{*}$ character.
The Dye-F $\mathbf{F}^{+}+\ldots \mathrm{Cl}^{-}$spectrum was calculated to have only two groups of transitions (Supplementary Fig. 7): A weak transition from the $\mathrm{Cl}^{-} 3 \mathrm{p}$ orbital to the phenyl $\pi^{*}$ orbitals, consistent with a weakly covalent $\pi$-anion interaction $\left(\pi^{*} \leftarrow \mathrm{Cl}_{1 \mathrm{~s}}\right)$; and the edge peak $\left(\mathrm{Cl}_{4 \mathrm{p}} \leftarrow \mathrm{Cl}_{1 \mathrm{~s}}\right)$. These results suggest that the small pre-edge peak observed for $\mathrm{Dye}^{-\mathrm{F}^{\bullet}+\ldots \mathrm{Cl}^{-}}$may arise from $\pi$-anion interactions, and not just $\mathrm{Cl}^{-}$interactions with the $\mathrm{TiO}_{2}$ substrate as we previously assumed ${ }^{37}$. Simulated XAS spectra of the analogous Dye- $\mathbf{I}^{\bullet}+\ldots \mathrm{Cl}^{-}{ }_{\pi \text {-anion }}$ and $\mathbf{D y e}-\mathrm{Br}^{\bullet}+\ldots \mathrm{Cl}^{-}{ }_{\pi \text {-anion }}$ structures shows only nominal differences in the XAS signal compared to Dye-F ${ }^{\bullet}+\ldots \mathrm{Cl}^{-}$(Supplemental Fig. 8). Considering that these $\pi$ anion interactions would be present to the same or lesser extent for the more polarizable halogen substituents compared to the Dye- $\mathrm{F}^{\bullet}+\ldots \mathrm{Cl}^{-}$control, they do not impact our conclusions.

TD-DFT methods are known to significantly underestimate the energy of XAS transitions as a result of the omission of occupied orbital relaxation in response to the core hole, the omission of relativistic stabilization, and other errors associated with the functional ${ }^{47-49}$. To correct for these errors, the energies of the XAS spectra were shifted by a fixed value until the calculated $\mathrm{Cl}_{4 \mathrm{p}} \leftarrow \mathrm{Cl}_{1 \mathrm{~s}}$ edge peak maximum matched the experimental edge peak maximum as described in the Supplementary Methods section. After correction, the calculated energies of the groups of transitions allow us to assign the pre-edge spectral features observed in the experimental Dye- $\mathbf{I}^{\bullet}+\ldots \mathrm{Cl}^{-}$and $\mathrm{Dye}-\mathrm{Br}^{\bullet}+\ldots \mathrm{Cl}^{-}$ XAS spectra. The calculated $\pi^{*} \leftarrow \mathrm{Cl}_{1 \mathrm{~s}}$ transitions appeared between 2817.4 and $2819.9 \mathrm{eV}$ for $\mathbf{D y e}-\mathrm{I}^{\bullet}+\ldots \mathrm{Cl}^{-}$and between 2817.0 and $2819.7 \mathrm{eV}$ for Dye- $\mathbf{B r}^{\bullet}+\ldots \mathrm{Cl}^{-}$(Table 1). The wellresolved pre-edge peaks in the experimental Dye- $\mathbf{I}^{\bullet}+\ldots \mathrm{Cl}^{-}$ and Dye- $\mathbf{B r}^{\bullet+}+\ldots \mathrm{Cl}^{-}$XAS spectra were observed at 2818.6 and $2818.4 \mathrm{eV}$, respectively, in good agreement with the calculated $\pi^{*} \leftarrow \mathrm{Cl}_{1 \mathrm{~s}}$ transitions. The $\sigma^{*} \leftarrow \mathrm{Cl}_{1 \mathrm{~s}}$ transitions were calculated to appear between 2822.0 and $2824.1 \mathrm{eV}$ for $\mathbf{D y e}-\mathbf{I}^{\bullet}+\ldots \mathrm{Cl}^{-}$and between 2822.1 and $2824.4 \mathrm{eV}$ for Dye- $\mathbf{B r}^{\circ}+\ldots \mathrm{Cl}^{-}$(Table 1). In even the most generous case, these transitions are too high in energy by several $\mathrm{eV}$ to account for the well-resolved pre-edge peak, and in many cases are unresolved from the edge peak (Supplementary Figs. 5 and 6). Instead, the $\sigma^{*} \leftarrow \mathrm{Cl}_{1 \mathrm{~s}}$ transitions are in better agreement with the pre-edge shoulder observed in the $2820-2 \mathrm{eV}$ range for both $\mathrm{Dye}-\mathrm{I}^{\bullet}+\ldots \mathrm{Cl}^{-}$and $\mathrm{Dye}-\mathrm{Br}^{\bullet}+\ldots \mathrm{Cl}^{-}$.

$\pi$-orbital covalency. As discussed above, the integrated intensity of a pre-edge peak in K-edge XAS spectroscopy is directly proportional to the degree of orbital mixing that gives rise to the peak $^{39,46}$. Bearing this in mind, the relative intensity of the observed $\pi^{*} \leftarrow \mathrm{Cl}_{1 \text { s }}$ pre-edge features compared to the edge peaks in the experimental Dye-I ${ }^{\bullet}+\ldots \mathrm{Cl}^{-}$and Dye- $\mathbf{B r}^{\bullet}+\ldots \mathrm{Cl}^{-}$XAS spectra suggests a significant amount of $\pi$-orbital covalency. The extent to which these pre-edge peak intensities-and by extension the $\pi$ orbital covalency-were reflected in the TD-DFT calculated XAS spectra was found to be very sensitive to the choice of functional (Fig. 4, Supplementary Figs. 5 and 6). Only the pure density functionals BP86 and M06-L and the low-HF exchange functional O3LYP predicted the $\pi^{*} \leftarrow \mathrm{Cl}_{1 \text { s }}$ pre-edge features to have any appreciable intensity compared to the edge peak. This result is not surprising because pure GGA and meta-GGA functionals are known to favor higher covalency in molecular systems ${ }^{46,50}$, and the BP86 functional in particular has been shown to better describe metal-ligand covalency in XAS spectra compared to hybrid functionals ${ }^{51}$. Electron density difference map (EDDM) plots of the $\pi^{*} \leftarrow \mathrm{Cl}_{1 \mathrm{~s}}$ transition with both Dye-I ${ }^{\bullet}+\ldots \mathrm{Cl}^{-}$and Dye$\mathrm{Br}^{*}+\ldots \mathrm{Cl}^{-}$reveal that mixing of the $\mathrm{Cl} 3 \mathrm{p}$ orbitals into the $\pi-$ orbitals of the dye decreases with the intensity of the pre-edge feature. The lowest energy empty orbital of the dimers is the single-electron $\beta$-spin LUMO which can be viewed as the empty 
a

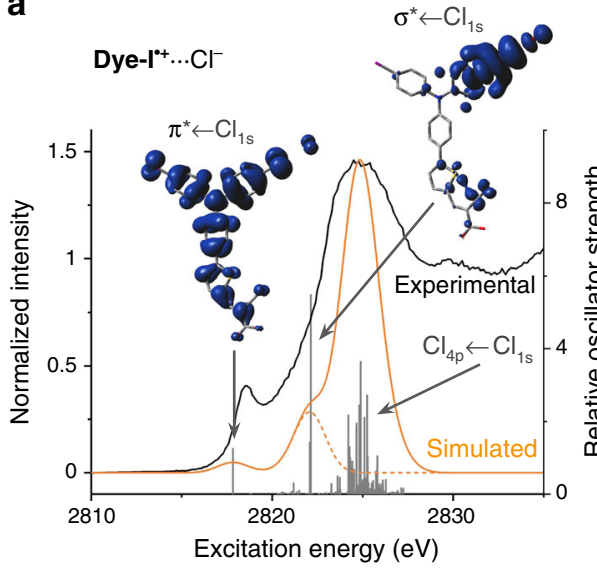

b

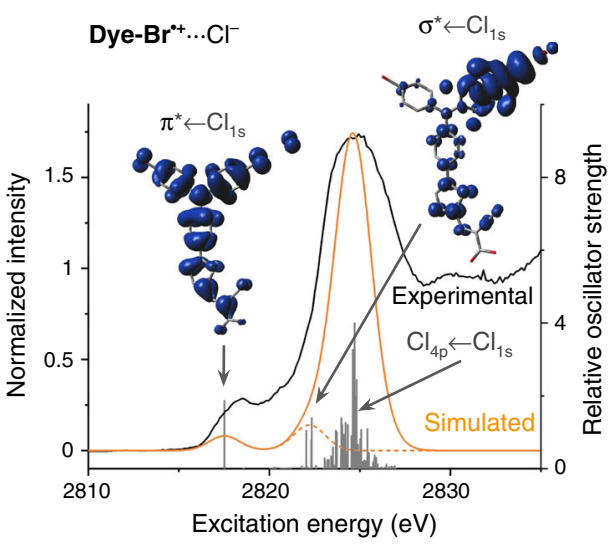

Fig. 3 Experimental and TD-DFT simulated XAS spectra. The experimental XAS spectra (black lines) of Dye-1 $\mathbf{I}^{\bullet+\ldots} \mathrm{Cl}^{-}(\mathbf{a})$ and $\mathbf{D y e}-\mathrm{Br}^{\bullet+\ldots} \mathrm{Cl}^{-}(\mathbf{b})$ and the corresponding TD-DFT simulated spectra (calculated using BP86, solid orange lines) are shown along with the relative oscillator strengths of the individual calculated transitions (vertical gray lines). The height of the simulated edge peaks were normalized to the height of the experimental edge peak. The transitions corresponding to pre-edge features were visualized as electron density difference maps (EDDMs) at an isovalue of 0.0004 (inset). The blue lobes represent positive changes in electron density corresponding to the acceptor orbital(s) of the transitions, while the donor orbital was always the chloride $1 \mathrm{~s}$ (indicated by a negative change in electron density in red and not always visible). In order to highlight the pre-edge features resulting from $\sigma$ and $\pi$-covalency in Dye-1 $\mathbf{I}^{\bullet} \ldots \mathrm{Cl}^{-}$and $\mathbf{D y e}-\mathrm{Br}^{\bullet}+\ldots \mathrm{Cl}^{-}$, truncated versions of the simulated spectra excluding the high energy $\mathrm{Cl}_{4 \mathrm{p}} \leftarrow \mathrm{Cl}_{1 \mathrm{~s}}$ edge peak transitions were also generated (dotted orange lines).

Table 1 Calculated energies of the major pre-edge transitions for $\mathrm{Dye}-\mathrm{I}^{\bullet}+\ldots \mathrm{Cl}^{-}$and $\mathrm{Dye}-\mathrm{Br}^{\bullet}+\ldots \mathrm{Cl}^{-}$.

\begin{tabular}{|c|c|c|c|c|}
\hline Functional & $\begin{array}{l}\text { Dye- } I^{\bullet}+\ldots \mathrm{Cl}^{-} \pi^{\star} \leftarrow \mathrm{Cl}_{1 \mathrm{~s}} \\
\text { transition energy }(\mathrm{eV})^{\mathrm{a}}\end{array}$ & $\begin{array}{l}\text { Dye-- }{ }^{\bullet}+\ldots \mathrm{Cl}^{-} \sigma^{\star} \leftarrow \mathrm{Cl}_{1 \mathrm{~s}} \\
\text { transition energy }(\mathrm{eV})^{\mathrm{a}}\end{array}$ & $\begin{array}{l}\text { Dye- } \mathrm{Br}^{\bullet}+\ldots \mathrm{Cl}^{-} \pi^{\star} \leftarrow \mathrm{Cl}_{1 \mathrm{~s}} \\
\text { transition energy }(\mathrm{eV})^{\mathrm{a}}\end{array}$ & $\begin{array}{l}\text { Dye- } \mathrm{Br}^{\bullet}+\ldots \mathrm{Cl}^{-} \sigma^{\star} \leftarrow \mathrm{Cl}_{1 \mathrm{~s}} \\
\text { transition energy }(\mathrm{eV})^{\mathrm{a}}\end{array}$ \\
\hline BP86 & 2817.83 & 2822.13 & 2817.52 & 2822.34 \\
\hline M06-L & 2817.38 & 2821.97 & 2816.97 & 2822.06 \\
\hline B3LYP & 2818.83 & 2823.13 & 2818.53 & 2823.38 \\
\hline PBEO & 2818.85 & 2823.12 & 2818.54 & 2823.41 \\
\hline M06 & 2819.6 & 2823.71 & 2819.27 & 2823.59 \\
\hline
\end{tabular}

half of the $\pi$-symmetric SOMO. The trend in $\pi^{*} \leftarrow \mathrm{Cl}_{1 \text { s }}$ intensities is mirrored by the $\mathrm{Cl}^{-}$involvement in the $\beta$-LUMO (Supplementary Figs. 9 and 10, Supplementary Tables 1 and 2).

While TD-DFT is generally the method of choice for accurately simulating K-edge XAS spectra ${ }^{47-49,51}$, the qualitative results are only as reliable as the underlying ground-state Kohn-Sham wavefunction. In order to verify the qualitative correctness of our TD-DFT models, we sought to examine the source of the wide discrepancy in predicted $\pi$-covalency from different DFT functionals. One possible source of this discrepancy is the selfinteraction error (SIE) inherent to DFT methods (caused by the unphysical repulsion of an electron by its own density) which is known to speciously favor electron delocalization ${ }^{52-57}$. Wavefunction methods based on Hartree-Fock theory do not suffer from SIE and can potentially provide more qualitatively reliable results, provided that electron correlation is accounted for in a sufficiently rigorous way. While open-shell systems are persistently a challenge for wavefunction methods, Neese et al. ${ }^{58}$ recently demonstrated an orbital optimized Møller-Plesset 2nd order perturbation (OOMP2) method that accurately reproduces a wide range of experimental results for organic radicals ${ }^{59}$. We therefore employed p-iodoaniline as a truncated model system to validate our results using this method (Fig. 5). This choice of model system was supported by the fact that both the TD-DFT XAS simulations and DFT calculated frontier MOs for the [piodoaniline $]^{\bullet+} \ldots \mathrm{Cl}^{-}$system show the same qualitative features and functional dependence as the full Dye- $\mathbf{I}^{\bullet}+\ldots \mathrm{Cl}^{-}$system (Supplementary Figs. 11 and 12). A comparable XAS simulation based on the OOMP2 wavefuntion was generated using the configuration interaction singles with MP2 doubles correction (CIS(D)) method. In this OOMP2-CIS(D) simulation, the $\pi^{*} \leftarrow \mathrm{Cl}_{1 \mathrm{~s}}$ preedge feature is still observed in the [p-iodoaniline ${ }^{\bullet+}+. \mathrm{Cl}^{-}$XAS spectrum, albeit at substantially lower energy and higher intensity relative to the TD-DFT results, and the frontier MOs reveal significant $\mathrm{Cl}^{-}$involvement in the $\beta$-LUMO (Fig. 5), in qualitative agreement with the DFT results. We can therefore conclude that $\pi$-covalency in the [p-iodoaniline $]^{\bullet+}+. \mathrm{Cl}^{-}$system-and by extension the Dye- $\mathbf{X}^{\bullet}+\ldots \mathrm{Cl}^{-}$systems-is not a result of SIE.

Closer examination of the Dye- $\mathbf{X}^{\bullet+}+. \mathrm{Cl}^{-}$electronic structures instead points to the large disparity in predicted $\pi$-covalency being a result of differences in the donor-acceptor orbital energy gap. Zhang and Musgrave have demonstrated that the calculated HOMO-LUMO gap of molecules is highly dependant on the choice of DFT functional, and according to their study hybrid functionals consistently overestimate this gap ${ }^{60}$. A similar effect is observed with our Dye- $\mathbf{X}^{\bullet+}+\mathrm{Cl}^{-}$systems, as illustrated by the 
a

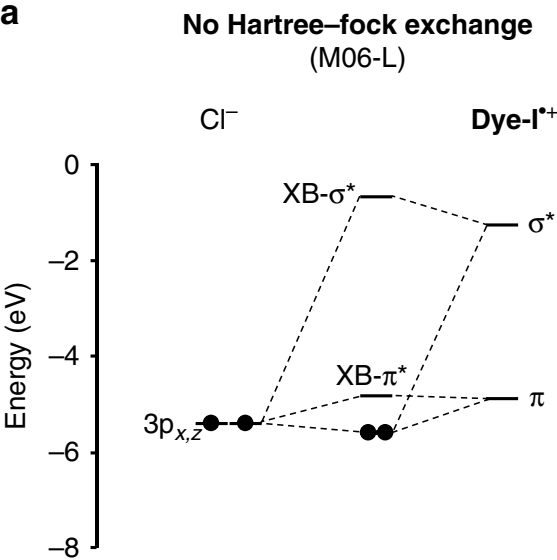

C

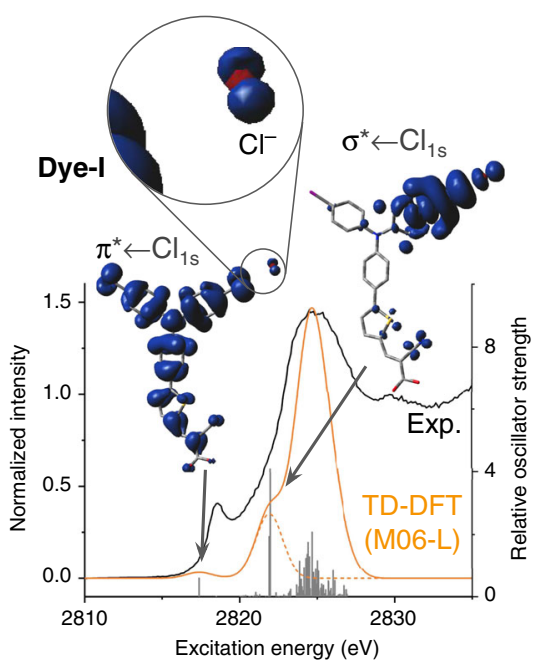

b High Hartree-fock exchange (M06-2X)

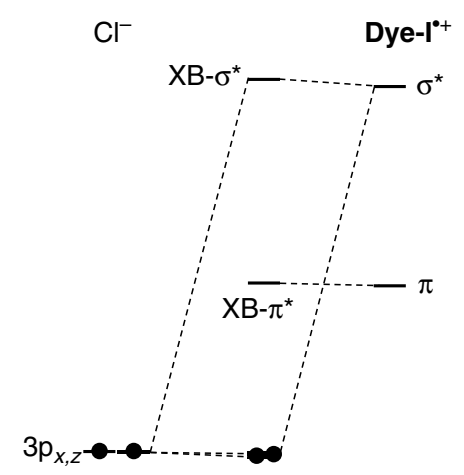

d

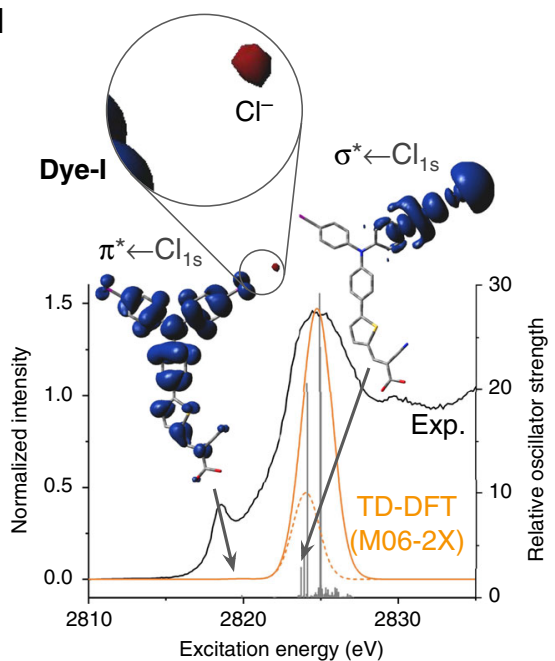

Fig. 4 DFT functional dependence of computational results with Dye-1•+...Cl-. a, b Molecular orbital diagrams describing the orbital mixing involved in the halogen bonding interaction between $\mathrm{Cl}^{-}$and $\mathbf{D y e}-\mathrm{I}^{\mathbf{\bullet}}$. These molecular orbital diagrams were generated from the $\beta$-spin single-electron Kohn-Sham orbital eigenvalues calculated using either the pure meta-GGA functional M06-L (a) or the high Hartree-Fock exchange hybrid functional M06-2X(b) and are simplified to consider only the orbital interactions resulting in the acceptor orbitals for the XAS pre-edge transitions. c, $\mathbf{d}$ TD-DFT simulated XAS spectra (orange lines) calculated using M06-L (c) or M06-2X(d) overlaid on the experimental Dye-1 ${ }^{\bullet}+\ldots \mathrm{Cl}^{-}$spectrum (black lines). The indicated pre-edge transitions are visualized with EDDMs as described in the Fig. 3 caption. In order to highlight the pre-edge features resulting from $\sigma$ - and $\pi$-covalency in Dye$\mathbf{I}^{\bullet+\ldots} \mathrm{Cl}^{-}$, truncated versions of the simulated spectrum excluding the high energy $\mathrm{Cl}_{4 \mathrm{p}} \leftarrow \mathrm{Cl}_{1 \mathrm{~s}}$ edge peak transitions were also generated (dotted orange line).

quantitative MO diagrams of the Dye- $\mathrm{I}^{\bullet}+\ldots \mathrm{Cl}^{-}$halogen bonding interaction constructed using each of the tested functionals (Supplementary Figs. 13-20). The orbital energy effect is exemplified by the Minnesota functionals (Fig. 4) where the energy of the empty $\pi$-symmetry Dye- $\mathbf{I}^{\bullet+}$ acceptor orbitals $\left(A_{\pi}\right)$ rises as a function of increasing exact HF exchange in the functional. In contrast, the filled $3 \mathrm{p}$ orbitals of the chloride nucleophile exhibit an inverse energy trend with respect to HF exchange character. As a result, the energy gap between the $3 \mathrm{p}$ donor orbitals on $\mathrm{Cl}^{-}$and the $A_{\pi}$ orbital on Dye- $\mathrm{I}^{\cdot+}$ is increased from $0.21 \mathrm{eV}$ for the GGA functional BP86 at one extreme to $3.89 \mathrm{eV}$ for the hybrid BH\&HLYP at the other extreme. Basic MO theory states that the degree of orbital mixing between two parent orbitals to form an $\mathrm{MO}$ is a function of the energy gap between the parent orbitals $^{61}$. On this basis, the differences in $\pi$-orbital covalency predicted by the various functionals under investigation are attributed entirely to the differences in the orbital energy gap.

\section{Discussion}

The existing model of covalency in halogen bonding only considers $\sigma$-symmetry donation of electron density from the nucleophile into the $\sigma^{*}$ orbital of the halogen bond donor $23,24,41,42$. Our study demonstrates that the observed preedge features in the experimental XAS spectra for Dye-I ${ }^{\bullet}+\ldots \mathrm{Cl}^{-}$ and Dye- $\mathrm{Br}^{\circ}+\ldots \mathrm{Cl}^{-}$can be attributed to a $\pi$-orbital interaction. While our computational models do predict an XAS transition corresponding to $\sigma$-covalency for both the Dye-I ${ }^{\bullet}+\ldots \mathrm{Cl}^{-}$and Dye$\mathrm{Br}^{\bullet}+\ldots \mathrm{Cl}^{-}$dimers, all of the methods we employ predict this transition to be too high in energy by at least $3 \mathrm{eV}$ relative to the edge peak to be resolved (Fig. 3, Supplementary Figs. 5 and 6). We instead propose that the shoulders experimentally observed in the $2820-2 \mathrm{eV}$ range are consequent of the $\sigma$-interactions. This conclusion is further supported by the calculated intensities of the $\sigma^{*} \leftarrow \mathrm{Cl}_{1 \text { s }}$ transition for both Dye-I ${ }^{\bullet}+\ldots \mathrm{Cl}^{-}$and $\mathbf{D y e}-\mathrm{Br}^{\bullet}+\ldots \mathrm{Cl}^{-}$ mirroring the relative prominence of this shoulder feature in the experimental spectra.

The $\pi^{*} \leftarrow \mathrm{Cl}_{1 \text { s }}$ pre-edge peak at $\sim 2818.5 \mathrm{eV}$ must therefore be the result of a much lower energy orbital. For both the Dye-I'+ and Dye-Br ${ }^{\circ}+\mathrm{XB}$-donors the lowest energy empty orbital is the $\pi$-symmetry $\beta$-LUMO that is orthogonal to the halogen bond axis. The lowest energy acceptor orbitals that can possibly result from these halogen bonds are therefore mixing of the $\mathrm{Cl}^{-} 3 \mathrm{p}$ 
a

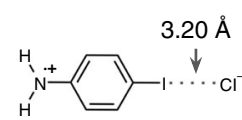

b

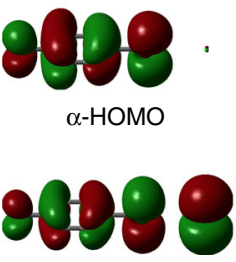

$\beta$-LUMO
C

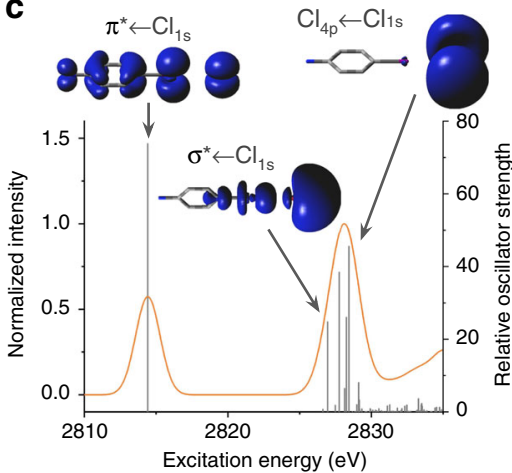

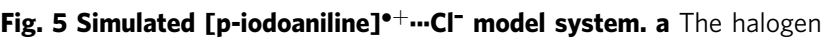
bonding adduct structure of [p-iodoaniline ${ }^{\bullet+\ldots} \mathrm{Cl}^{-}$. $\mathbf{b}$ The $\alpha$ - and $\beta$-spin single-electron orbital components of the [p-iodoaniline $]^{\bullet+. .} \mathrm{Cl}^{-}$SOMO, the $\alpha-\mathrm{HOMO}$ and $\beta$-LUMO, calculated at the OOMP2 level and plotted at an isovalue of 0.002. c The configuration interaction singles with MP2 doubles correction (CIS(D)) simulated XAS spectrum for [p-iodoaniline $]^{\bullet+. .} \mathrm{Cl}^{-}$ (calculated using OOMP2, orange line) is shown along with the relative oscillator strengths of the individual calculated transitions (vertical gray lines). The height of the simulated edge peaks was normalized to 1 . The transitions corresponding to pre-edge and edge peak features were visualized as EDDMs at an isovalue of 0.0008 (inset). The blue lobes represent positive changes in electron density corresponding to the acceptor orbital(s) of the transitions, while the donor orbital was always the chloride 1s (indicated by a negative change in electron density in red and not always visible).

orbitals into this $\beta$-LUMO. Given the symmetry of the orbitals involved and the linear geometry of the halogen bond, this interaction must also have $\pi$-symmetry analogous to the schematic in Fig. 1. Only those computational methods that account for $\pi$-orbital covalency correctly predicted the pre-edge features of the Dye- $\mathrm{I}^{\bullet}+\ldots \mathrm{Cl}^{-}$and Dye- $\mathrm{Br}^{\bullet}+\ldots \mathrm{Cl}^{-}$experimental XAS spectra.

This study provides an expanded description of the halogen bond. The standard covalent model of the halogen bonding interaction describes $\sigma$-symmetric orbital overlap, but we show that $\pi$-symmetric overlap is also possible and can have a significant impact on the observable electronic properties of halogen bonded molecules. We demonstrate this effect using a radical cation XB-donor and it remains to be seen how generalizable it is. Nevertheless, we believe that this effect can be extended beyond open-shell systems, such as in the situation described by Supplementary Fig. 1c. In much the same way as metal coordination bonds can contain a $\pi$-covalent component, $\pi$-covalency in halogen bonds should be considered whenever the nucleophile and electrophile have empty and filled $\pi$-symmetric orbitals that are close in energy. The majority of hybrid DFT functionals commonly used to model halogen bonding significantly underestimate this $\pi$-covalency. This broadened description of the halogen bond highlights new opportunities to design XB-donors and acceptors with the ability to form intermolecular $\pi$ interactions. Halogen bonds could thereby be used to modify reactivity and tune electronic properties in ways that cannot be achieved by other intermolecular interactions.

\section{Data availability}

Supplementary Figures, Supplementary Methods and Supplementary Tables are included in the Supplementary Information file. DFT optimized molecular geometries are reported in the Supplementary Data 1 file. Additional data supporting these findings are available from the corresponding author upon request. Source data are provided with this paper.

Received: 12 February 2020; Accepted: 9 June 2020;

Published online: 03 July 2020

\section{References}

1. Hassel, O. Structural aspects of interatomic charge-transfer bonding. Science 170, 497-502 (1970).

2. Legon, A. C. Prereactive complexes of dihalogens XY with lewis bases B in the gas phase: a systematic case for the halogen analogue B..XY of the hydrogen bond B...HX. Angew. Chem. Int. Ed. 38, 2686-2714 (1999).

3. Legon, A. C. The halogen bond: an interim perspective. Phys. Chem. Chem. Phys. 12, 7736-7747 (2010).

4. Wolters, L. P. \& Bickelhaupt, F. M. Halogen bonding versus hydrogen bonding: a molecular orbital perspective. ChemistryOpen 1, 96-105 (2012).

5. Politzer, P., Murray, J. S. \& Clark, T. Halogen bonding: an electrostaticallydriven highly directional noncovalent interaction. Phys. Chem. Chem. Phys. 12, 7748-7710 (2010).

6. Metrangolo, P., Neukirch, H., Pilati, T. \& Resnati, G. Halogen bonding based recognition processes: a world parallel to hydrogen bonding. Acc. Chem. Res. 38, 386-395 (2005).

7. Cavallo, G. et al. The halogen bond. Chem. Rev. 116, 2478-2601 (2016)

8. Pimentel, G. C. \& McClellan, A. L. The Hydrogen Bond (W.H. Freeman \& Co. San Francisco, 1960).

9. Corradi, E., Meille, S. V., Messina, M. T., Metrangolo, P. \& Resnati, A. G. Halogen bonding versus hydrogen bonding in driving self-assembly processes. Angew. Chem. Int. Ed. 39, 1782-1786 (2000).

10. Buckingham, A. D., Del Bene, J. E. \& McDowell, S. A. C. The hydrogen bond. Chem. Phys. Lett. 463, 1-10 (2008).

11. Bulfield, D. \& Huber, S. M. Halogen bonding in organic synthesis and organocatalysis. Chem. Eur. J. 22, 14434-14450 (2016).

12. Metrangolo, P., Meyer, F., Pilati, T., Resnati, G. \& Terraneo, G. Halogen bonding in supramolecular chemistry. Angew. Chem. Int. Ed. 47, 6114-6127 (2008).

13. Berger, G., Soubhye, J. \& Meyer, F. Halogen bonding in polymer science: from crystal engineering to functional supramolecular polymers and materials. Polym. Chem. 6, 3559-3580 (2015).

14. Persch, E., Dumele, O. \& Diederich, F. Molecular recognition in chemical and biological systems. Angew. Chem. Int. Ed. 54, 3290-3327 (2015).

15. Wilcken, R., Zimmermann, M. O., Lange, A., Joerger, A. C. \& Boeckler, F. M. Principles and applications of halogen bonding in medicinal chemistry and chemical biology. J. Med. Chem. 56, 1363-1388 (2013).

16. Politzer, P., Lane, P., Concha, M. C., Ma, Y. \& Murray, J. S. An overview of halogen bonding. J. Mol. Model. 13, 305-311 (2007).

17. Clark, T., Hennemann, M., Murray, J. S. \& Politzer, P. Halogen bonding: the $\sigma$-hole: Proceedings of 'Modeling interactions in biomolecules II', Prague, September 5th-9th, 2005. J. Mol. Model. 13, 291-296 (2007).

18. Koláŕ, M. H. \& Hobza, P. Computer modeling of halogen bonds and other $\sigma$ hole interactions. Chem. Rev. 116, 5155-5187 (2016).

19. Pauling, L. The shared-electron chemical bond. Proc. Natl Acad. Sci. USA 14, 359-362 (1928).

20. Pauling, L. The nature of the chemical bond. Application of results obtained from the quantum mechanics and from a theory of paramagnetic susceptibility to the structure of molecules. J. Am. Chem. Soc. 53, 1367-1400 (1931).

21. Gilli, G. \& Gilli, P. The Nature of the Hydrogen Bond (Oxford University Press, New York, 2009).

22. Grabowski, S. J. What is the covalency of hydrogen bonding? Chem. Rev. 111 2597-2625 (2011)

23. Oliveira, V., Kraka, E. \& Cremer, D. The intrinsic strength of the halogen bond: electrostatic and covalent contributions described by coupled cluster theory. Phys. Chem. Chem. Phys. 18, 33031-33046 (2016).

24. Angarov, V. \& Kozuch, S. On the $\sigma, \pi$ and $\delta$ hole interactions: a molecular orbital overview. New J. Chem. 42, 1413-1422 (2018).

25. Wang, C., Danovich, D., Mo, Y. \& Shaik, S. On the nature of the halogen bond. J. Chem. Theory Comput. 10, 3726-3737 (2014).

26. Rosokha, S. V., Stern, C. L. \& Ritzert, J. T. Experimental and computational probes of the nature of halogen bonding: complexes of bromine-containing molecules with bromide anions. Chem. Eur. J. 19, 8774-8788 (2013).

27. Rosokha, S. V. Electron-transfer reactions of halogenated electrophiles: a different look into the nature of halogen bonding. Faraday Discuss. 203, 315-332 (2017).

28. Mulliken, R. S. Molecular compounds and their spectra. II. J. Am. Chem. Soc. 74, 811-824 (1952).

29. Mulliken, R. S. Molecular compounds and their spectra. III. The interaction of electron donors and acceptors. J. Phys. Chem. 56, 801-822 (1952). 
30. Politzer, P., Murray, J. S. \& Clark, T. Mathematical modeling and physical reality in noncovalent interactions. J. Mol. Model. 21, 52 (2015).

31. Clark, T. Polarization, donor-acceptor interactions, and covalent contributions in weak interactions: a clarification. J. Mol. Model. 23, 297 (2017).

32. Walton, J. R., Rivera-Rivera, L. A., Lucchese, R. R. \& Bevan, J. W. Is there any fundamental difference between ionic, covalent, and others types of bond? A canonical perspective on the question. Phys. Chem. Chem. Phys. 19, 15864-15869 (2017).

33. Jenkins, Z. Do you need to believe in orbitals to use them?: Realism and the autonomy of chemistry. Philos. Sci. 70, 1052-1062 (2003).

34. Ang, S. J., Mak, A. M. \& Wong, M. W. Nature of halogen bonding involving $\pi$-systems, nitroxide radicals and carbenes: a highlight of the importance of charge transfer. Phys. Chem. Chem. Phys. 20, 26463-26478 (2018).

35. Swords, W. B. et al. Evidence for interfacial halogen bonding. Angew. Chem. Int. Ed. 55, 5956-5960 (2016).

36. Simon, S. J. C. et al. Halogen bonding promotes higher dye-sensitized solar cell photovoltages. J. Am. Chem. Soc. 138, 10406-10409 (2016).

37. Parlane, F. G. L. et al. Spectroscopic detection of halogen bonding resolves dye regeneration in the dye-sensitized solar cell. Nat. Commun. 8, 1761 (2017).

38. Glaser, T., Hedman, B., Hodgson, K. O. \& Solomon, E. I. Ligand K-edge X-ray absorption spectroscopy: a direct probe of ligand-metal covalency. Acc. Chem. Res. 33, 859-868 (2000).

39. Shadle, S. E., Hedman, B., Hodgson, K. O. \& Solomon, E. I. Ligand K-edge Xray absorption spectroscopy as a probe of ligand-metal bonding: charge donation and covalency in copper-chloride systems. Inorg. Chem. 33, 4235-4244 (1994).

40. Solomon, E. I., Hedman, B., Hodgson, K. O., Dey, A. \& Szilagyi, R. K. Ligand K-edge X-ray absorption spectroscopy: covalency of ligand-metal bonds. Coord. Chem. Rev. 249, 97-129 (2005).

41. Mustoe, C. L., Gunabalasingam, M., Yu, D., Patrick, B. O. \& Kennepohl, P. Probing covalency in halogen bonds through donor K-edge X-ray absorption spectroscopy: polyhalides as coordination complexes. Faraday Discuss. 203, 79-91 (2017)

42. Robinson, S. W. et al. Evidence for halogen bond covalency in acyclic and interlocked halogen-bonding receptor anion recognition. J. Am. Chem. Soc 137, 499-507 (2015).

43. Hagopian, L., Koehler, G. \& Walter, R. I. Substituent effects on the properties of stable aromatic free radicals. Oxidation-reduction potentials of triarylamine-triarylaminium ion systems. J. Phys. Chem. 71, 2290-2296 (1967).

44. Amthor, S., Noller, B. \& Lambert, C. UV/Vis/NIR spectral properties of triarylamines and their corresponding radical cations. Chem. Phys. 316, 141-152 (2005).

45. Kellett, C. W., Swords, W. B., Turlington, M. D., Meyer, G. J. \& Berlinguette, C. P. Resolving orbital pathways for intermolecular electron transfer. Nat. Commun. 9, 4916 (2018).

46. Gewirth, A. A., Cohen, S. L., Schugar, H. J. \& Solomon, E. I. Spectroscopic and theoretical studies of the unusual EPR parameters of distorted tetrahedral cupric sites: correlations to $\mathrm{x}$-ray spectral features of core levels. Inorg. Chem. 26, 1133-1146 (1987).

47. Ray, K., Debeer George, S., Solomon, E. I., Wieghardt, K. \& Neese, F. Description of the ground-state covalencies of the bis(dithiolato) transition metal complexes from X-ray absorption spectroscopy and time-dependent density-functional calculations. Chemistry 13, 2783-2797 (2007).

48. Kozimor, S. A. et al. Trends in covalency for $\mathrm{d}$ - and f-element metallocene dichlorides identified using chlorine K-edge X-ray absorption spectroscopy and time-dependent density functional theory. J. Am. Chem. Soc. 131, 12125-12136 (2009).

49. Minasian, $\mathrm{S}$. G. et al. Determining relative $\mathrm{f}$ and $\mathrm{d}$ orbital contributions to $\mathrm{M}$ $\mathrm{Cl}$ covalency in $\mathrm{MCl}_{6}{ }^{(2-)}(\mathrm{M}=\mathrm{Ti}, \mathrm{Zr}, \mathrm{Hf}, \mathrm{U})$ and $\mathrm{UOCl}_{5}{ }^{(-)}$using $\mathrm{Cl} \mathrm{K}$-edge $\mathrm{X}$-ray absorption spectroscopy and time-dependent density functional theory. J. Am. Chem. Soc. 134, 5586-5597 (2012).

50. Neese, F. Theoretical study of ligand superhyperfine structure. Application to Cu(II) complexes. J. Phys. Chem. A 105, 4290-4299 (2001).

51. DeBeer George, S., Petrenko, T. \& Neese, F. Time-dependent density functional calculations of ligand K-edge X-ray absorption spectra. Inorg. Chim. Acta 361, 965-972 (2008).

52. Perdew, J. P. \& Zunger, A. Self-interaction correction to density-functional approximations for many-electron systems. Phys. Rev. B Condens. Matter 23, 5048-5079 (1981).

53. Mori-Sánchez, P., Cohen, A. J. \& Yang, W. Many-electron self-interaction error in approximate density functionals. J. Chem. Phys. 125, 201102 (2006).

54. Lundberg, M. \& Siegbahn, P. E. M. Quantifying the effects of the selfinteraction error in DFT: when do the delocalized states appear? J. Chem. Phys. 122, 224103 (2005).
55. Cohen, A. J., Mori-Sánchez, P. \& Yang, W. Challenges for density functional theory. Chem. Rev. 112, 289-320 (2012).

56. Hait, D. \& Head-Gordon, M. Delocalization errors in density functional theory are essentially quadratic in fractional occupation number. J. Phys. Chem. Lett. 9, 6280-6288 (2018).

57. Zhang, Y. \& Yang, W. A challenge for density functionals: Self-interaction error increases for systems with a noninteger number of electrons. J. Chem. Phys. 109, 2604-2608 (1998).

58. Neese, F., Schwabe, T., Kossmann, S., Schirmer, B. \& Grimme, S. Assessment of orbital-optimized, spin-component scaled second-order many-body perturbation theory for thermochemistry and kinetics. J. Chem. Theory Comput. 5, 3060-3073 (2009).

59. Sandhoefer, B., Kossmann, S. \& Neese, F. Derivation and assessment of relativistic hyperfine-coupling tensors on the basis of orbital-optimized second-order Møller-Plesset perturbation theory and the second-order Douglas-Kroll-Hess transformation. J. Chem. Phys. 138, 104102 (2013).

60. Zhang, G. \& Musgrave, C. B. Comparison of DFT methods for molecular orbital eigenvalue calculations. J. Phys. Chem. A 111, 1554-1561 (2007).

61. Albright, T. A., Burdett, J. K. \& Whangbo, M.-H. Orbital interactions in chemistry. (John Wiley and Sons, Hoboken, 2013).

\section{Acknowledgements}

We would like to thank Fraser G.L. Parlane and Dr. Phil A. Schauer at the University of British Columbia for their assistance in preparing the manuscript. We are grateful to the Canadian Natural Science and Engineering Research Council (RGPIN 337345-13 and RGPIN 2016-05453), Canadian Foundation for Innovation (229288), Canadian Institute for Advanced Research (BSE-BERL-162173), and Canada Research Chairs for financial support. This work is partially based on X-ray absorption spectra collected by Fraser G.L. Parlane and Pierre Kennepohl at the Stanford Synchrotron Radiation Lightsource (SSRL) at the SLAC National Accelerator Laboratory. The use of SSRL is supported by the U.S Department of Energy, Office of Science, and Office of Basic Energy Sciences under contract no. DE-AC02-76SF00515.

\section{Author contributions}

C.W.K., P.K., and C.P.B. designed the experiment. C.W.K. performed the computationa analysis. C.W.K. and C.P.B. wrote the manuscript and all authors contributed to discussing and editing the content.

\section{Competing interests}

The authors declare no competing interests.

\section{Additional information}

Supplementary information is available for this paper at https://doi.org/10.1038/s41467 020-17122-7.

Correspondence and requests for materials should be addressed to C.P.B.

Peer review information Nature Communications thanks the anonymous reviewers for their contribution to the peer review of this work.

Reprints and permission information is available at http://www.nature.com/reprints

Publisher's note Springer Nature remains neutral with regard to jurisdictional claims in published maps and institutional affiliations.

Open Access This article is licensed under a Creative Commons Attribution 4.0 International License, which permits use, sharing, adaptation, distribution and reproduction in any medium or format, as long as you give appropriate credit to the original author(s) and the source, provide a link to the Creative Commons license, and indicate if changes were made. The images or other third party material in this article are included in the article's Creative Commons license, unless indicated otherwise in a credit line to the material. If material is not included in the article's Creative Commons license and your intended use is not permitted by statutory regulation or exceeds the permitted use, you will need to obtain permission directly from the copyright holder. To view a copy of this license, visit http://creativecommons.org/ licenses/by/4.0/

(C) The Author(s) 2020 